\title{
Clinical Features, Treatment and Outcomes of Patients with Gestational Trophoblastic Neoplasm: An Experience from a Tertiary Center for Cancer Care in Thailand
}

\author{
Siriwan Tangiitgamol* and Sunamchok Srijaipracharoen \\ Department of Obstetrics and Gynecology, Faculty of Medicine Vajira Hospital, Navamindradhiraj University, Bangkok, Thailand
}

\begin{abstract}
Objectives: To assess clinical features, treatment and oncologic outcomes of patients with gestational trophoblastic neoplasm (GTN).

Methods: Patients with GTN who were treated in the institution during January 2000 to December 2018 were identified. Data collected were age at GTN diagnosis, index pregnancy, interval prior to GTN, FIGO stage, WHO score, treatment and outcomes.

Results: Total of 74 patients with available data were included in the study. Median age was 33 years (range, 17-52 years). The most common antecedent pregnancy was molar pregnancy (85.1\%). Stage I disease was most common (73.0\%). Majority were classified as low risk (85.1\%). All patients with metastases had pulmonary lesions. Other metastatic sites were brain, abdominal wall, vulva, and colon. Hysterectomy was performed in $17.8 \%$ as primary or interval surgery after few cycles of chemotherapy. Chemotherapy was given in $93.2 \%$ of patients, with methotrexate as the most common first-line drug (82.5\%). Response rate among patients who completed first-line chemotherapy was $83.8 \%$. The patients who had persistence or rising of serum B-hCG (14.7\%) or experienced chemotherapy side-effects (1.5\%) as well as those who had recurrence (6.8\%) had second-line chemotherapy, with few had further-line drugs. After a median follow-up of 22.7 months (range, $0.37-$ 210.9 months), only 1 patient was dead from progressive diseases. The others were alive and doing well without any evidences of diseases.
\end{abstract}

Conclusions: Majority of GTN had antecedent molar pregnancy, stage I and low risk disease. Surgery had certain role in selected patients. Chemotherapy was the mainstay of treatment yielding good oncologic outcomes.

\section{Introduction}

World Health Organization (WHO) which categorized tumors by histomorphology has divided gestational trophoblastic neoplasms (GTN) into choriocarcinoma, placental site trophoblastic tumor (PSTT), and epithelioid trophoblastic tumor (ETT) [1]. Although invasive mole is classified under the category of molar pregnancies [1], it is generally included in the reports of GTN due to its clinical behavior and treatment as a neoplasm.

A diagnosis of GTN is made by persistence or rising level of serum human chorionic gonadotropin (hCG) after termination of molar or other pregnancies whereas stage of disease is assigned according to the International Federation of Gynecology and Obstetrics (FIGO) based on anatomical location of diseases [2]. Along with the stage, risk score which is calculated from the sum score of several clinical features is also presented to categorize the patients into low-, high-, or ultra-high risk. Chemotherapy is the mainstay of treatment for GTN. Option of chemotherapy regimen, either single or combination drugs, is given according to the risk groups [2]. Surgical treatment may occasionally be used in selected patients with localized lesion $[3,4]$ or when clinically indicated, such as, bleeding [3,5,6].

Few authors raised some issues of management for GTN patients regarding the role of re-curettage after diagnosis [7], pattern or level of B-hCG necessitating chemotherapy after molar suction [8], requirement of chemotherapy after total surgical resection of lesions [9], the most effective regimen of chemotherapy $[10,11]$, and etc.
Because GTN is unevenly distributed globally, with higher frequency in Asian than Western countries, data from each region or country are important for an advance in knowledge regarding its presentation, clinical course, and outcomes after treatment of this uncommon disease. This study aimed to evaluate clinical features, treatment and outcomes of the patients with GTN. Reproductive outcomes after treatment were also assessed and would be reported elsewhere.

\section{Materials and methods}

A list of patients who had diagnoses invasive mole and GTN during January 2000 to December 2018 were searched from the hospital database using ICD10 codes i.e. D39.2 and M9100/1 (invasive mole), 9100/3 (choriocarcinoma), placental site trophoblastic tumor (9104/1), and 9105/3 (epithelioid trophoblastic tumor). Additional numbers of patients were searched from the archive of the Gynecologic Oncology Unit, Department of Obstetrics and Gynecology of our institution. Exclusion criteria were patients who had no available medical records.

A general practice in our institution for GTN patients generally followed the international guidelines for diagnosis and management of

${ }^{\star}$ Correspondence to: Siriwan Tangjitgamol, 681 Samsen road, Dusit district, Bangkok, Thailand, E-mail: siriwanonco@nmu.ac.th; siriwanonco@yahoo.com

Key words: gestational trophoblastic neoplasm, treatment, oncologic outcomes

Received: April 01, 2020; Accepted: April 10, 2020; Published: April 15, 2020 
GTD which have been released continually $[2,12,13]$. The diagnosis was made when there was a plateau of serum B-hCG levels for 3 consecutive weeks or longer, a 10\% rise in 2 consecutive weeks, persistence of B-hCG for 6 months or longer, or a histologic diagnosis of choriocarcinoma. Once a diagnosis of GTN was made, a protocol of metastatic survey was performed including complete physical examination, laboratory investigations including serum B-hCG level, biochemical study, urinalysis, and imaging studies. Imaging studies included pelvic ultrasound and chest $\mathrm{x}$-ray. Computerized tomography scans (CTscan) of whole abdomen, chest and brain were selectively performed when clinically indicated. The patient was staged and categorized into low risk (LR) or high risk (HR) including ultra-HR groups according to FIGO stage and WHO classification respectively.

Treatment, in general, was chemotherapy which was given until remission (defined as a decline of hCG level to $<10 \mathrm{mIU} / \mathrm{ml}$ ) plus additional courses in selected patients according to their risk. The regimen of chemotherapy was based on the risk score group. Surgical treatment was considered according to various factors e.g. the extent (focal vs multi-foci) and features of lesions (intact vs hemorrhage), age (young vs peri- or post-menopause) and desire for fertility (complete vs incomplete childbearing), the physician's preference, and the patient's decision.

Data collected were: age and date at GTN diagnosis, index pregnancies and interval before the diagnosis, FIGO stage, WHO score, date and type of treatment, histopathology if available, response, side effects, reasons for further lines of chemotherapy beyond the first line treatment, date of last follow-up and living status. Pertinent medical history particularly history of gestational trophoblastic diseases was also recorded.

Data were analyzed using SPSS statistical software, version 22 (IBM Corporation, Armonk, NY, USA). Descriptive statistics were used to analyze demographic data and were summarized as numbers with percentage, mean with standard deviation (SD) or median with range. Overall survival (OS) was obtained from date of diagnosis to date of death or last evaluation in patients who were alive. Characteristics features of index pregnancy, its interval before GTN diagnosis, and FIGO stage between the patients with LR or HR/ultra-HR were compared using chi-Square or Fisher Exact tests as appropriate. P-value $<0.05$ was considered significant.

\section{Results}

During the study period, a total of 74 patients who had a diagnosis of GTN and with available data were included in the study. The median age of patients at their GTN diagnosis was 33 years (range, 17-52 years), with 56 patients aged $\leq 40$ years $(75.7 \%)$. The most common index pregnancy was molar pregnancy (63 patients; $85.1 \%$ ); one was repeated molar pregnancy. All patients had abnormal B-hCG level: 54 patients $(73.0 \%)$ presented with abnormal serology with or without uterine lesions whereas the other 20 patients $(27.0 \%)$ had evidence of metastatic lung lesions ( 2 of them also had vulva and colon, or abdominal wall metastasis). Characteristic features of the GTN patients are shown in Table 1 .

The median duration from an index pregnancy to GTN was 1.5 months (range, 0.27-403.5 months). On the other hand, majority (84.3\%) had a diagnosis of GTN within 4 months after index pregnancy. Of note, 3 patients had interval of over 10 years before the diagnosis of GTN (2 terms, 1 abortion).
One patient had prior hysterectomy elsewhere for a diagnosis of abortion 10 years earlier. Out of 73 patients, 14 patients (19.2\%) had hysterectomy in the primary setting: $8(11.0 \%)$ had primary hysterectomy at the time of diagnosis whereas the other $6(8.2 \%)$ had interval surgery after slow response to few cycles of chemotherapy. Five patients also had resection of lesions (6 operative procedures) from uterus, abdominal wall, vulva or adnexa. Table 2 shows the types of primary surgery and pathology obtained.

Majority of patients had FIGO stage I disease (54 patients, 73.0\%) and were classified as low risk (63 patients, $85.1 \%$ ). The associations between the type of index pregnancy, its interval to GTN, stage and GTN risk group were studied. Non-molar pregnancies, interval $>4$ months, stage III-IV were significantly associated with HR/ ultra-HR GTN (Table 3). We also explored the association between the type of pregnancy with interval and stage of GTN. Non-molar pregnancy was

Table 1. Characteristic features of the patients with gestational trophoblastic neoplasia $(\mathrm{N}=74)$

\begin{tabular}{|l|c|c|}
\hline Characteristic features & N & \% \\
\hline Age at GTN diagnosis in years, median (range) & \multicolumn{2}{|c|}{33 (17-52) } \\
\hline$<40$ years & 56 & 75.7 \\
\hline$\geq 40$ years & 18 & 24.3 \\
\hline Type of index pregnancy* & & \\
\hline Molar pregnancy & 63 & 85.1 \\
\hline Abortion & 5 & 6.8 \\
\hline Term pregnancy & 4 & 5.4 \\
\hline Ectopic pregnancy & 2 & 2.7 \\
\hline Means of GTN diagnosis & & \\
\hline Persistence or elevation of B-hCG only & 25 & 33.8 \\
\hline Persist/ rising with intrauterine lesions & 29 & 39.2 \\
\hline Persist/ rising with metastatic lesions & 20 & 27.0 \\
\hline
\end{tabular}

*Including 8 patients who were diagnosed as invasive mole at diagnosis

Table 2. Surgical treatment and pathology at the primary treatment of gestational trophoblastic neoplasia $(\mathrm{N}=18)$

\begin{tabular}{|l|c|c|c|}
\hline \multirow{2}{*}{ Surgical treatment } & \multicolumn{3}{c|}{ Pathology, n (\%) } \\
\cline { 2 - 4 } & $\begin{array}{c}\text { No } \\
\text { pathology }\end{array}$ & $\begin{array}{c}\text { Invasive } \\
\text { mole }\end{array}$ & Choriocarcinoma \\
\hline $\begin{array}{l}\text { Primary hysterectomy with or without SO } \\
(\mathrm{n}=8)\end{array}$ & - & $7(87.5)$ & $1(12.5)$ \\
\hline $\begin{array}{l}\text { Interval hysterectomy with or without SO } \\
(\mathrm{n}=6)\end{array}$ & - & $2(33.3)$ & $4 *(66.7)$ \\
\hline Mass resection/ biopsy/ curettage (n=6) & $1 *(16.7)$ & - & $5(83.3)$ \\
\hline
\end{tabular}

*One patient had interval hysterectomy then subsequent salpingo-oophorectomy in another episode

Abbreviation: SO, salpingo-oophorectomy

Table 3. Risk score group according to features of patients with gestational trophoblastic neoplasia $(\mathrm{N}=74)$

\begin{tabular}{|l|c|c|c|}
\hline \multirow{2}{*}{$\begin{array}{l}\text { Features of pregnancy and stage } \\
\text { of GTN (n) }\end{array}$} & $\begin{array}{c}|c| \\
\text { Low-risk, } \\
\mathbf{N = 6 3} \\
\mathbf{n}(\mathbf{\%})\end{array}$ & $\begin{array}{c}\text { High- or ultra-high } \\
\text { risk, N=11 } \\
\mathbf{n}(\%)\end{array}$ & \multirow{2}{*}{ p-value } \\
\hline Preceding pregnancy & & & \\
\hline Molar pregnancy (n=63) & $59(93.7)$ & $4(6.3)$ & $<0.001$ \\
\hline Non-molar pregnancy (n=11) & $4(36.4)$ & $7(63.6)$ & \\
\hline Interval between pregnancy and GTN & & & 0.001 \\
\hline$<4$ months (n=63) & $58(92.1)$ & $5(7.9)$ & \\
\hline$\geq 4$ months (n=11) & $5(45.5)$ & $6(54.5)$ & \\
\hline FIGO stage of GTN & & & 0.036 \\
\hline Stage I* (n=54) & $49(90.7)$ & $5(9.3)$ & \\
\hline Stage III-IV (n=20) & $14(70.0)$ & $6(30.0)$ & \\
\hline
\end{tabular}

*All 8 patients with invasive mole were in stage I ( 6 were in low risk group and 2 in high risk group) 
more frequently associated with interval $>4$ months and stage III-IV than molar pregnancy: $54.5 \%$ vs $7.9 \%(\mathrm{p}=0.001)$ and $45.5 \%$ vs $23.8 \%$ $(\mathrm{p}=0.155)$, respectively.

Clinical features of 14 patients who had hysterectomy were studied. These patients were significantly older and had HR score more frequently than the others: median age of 45.0 years (range, 2752 years) vs 31.0 years (range, $17-50$ years) $(\mathrm{p}<0.001)$ with $50.0 \%(4 / 8$ patients) vs $14.3 \%$ ( $9 / 63$ patients) $(\mathrm{p}=0.031)$ in HR group. Although the patients with stage I diseases underwent hysterectomy more frequently than those with stage III/IV, 20.4\% (11/54 patients) compared to $15.0 \%$ ( $3 / 20$ patients), the difference was not statistically significant $(p=0.746)$.

After GTN diagnosis, 5 patients (6.8\%) in stage I (3 HR and 2 LR) did not have any chemotherapy. Four of whom underwent primary hysterectomy ( 3 invasive moles, scores 5,6,11 and 1 choriocarcinoma, score 8 ) and one had only uterine curettage (choriocarcinoma, score 7). All had serum B-hCG declined to normal after the operative procedures.

Among 69 patients who received chemotherapy, 61 had single agent and 8 had combined drugs. Almost all LR patients received single drug, with methotrexate as the most common agent (82.5\%). We explored the reasons why 3 patients in LR group had combined drugs (4.1\% of all patients). Two patients (stage III: score 2 and score 6 ) had their first cycle of chemotherapy elsewhere before referral to our service, so the same chemotherapy regimens (EMA or EMA-CO) were continued. Another patient (stage I: score 6) had EMA-CO for her choriocarcinoma perforating through uterine serosa.

The median cycle of first-line chemotherapy was 5 cycles (range, 1-12 cycles). Fewer chemotherapy cycles were required among patients who had hysterectomy than the others: 3.5 cycles (range 0-7 cycles) vs 5.0 cycles (range $0-12$ cycles) $(\mathrm{p}=0.080)$. Regarding the side effects of treatment, only one HR patient experienced respiratory distress syndrome after the second cycle of EMA-CO. Her condition was resolved with supportive treatment before continuing with $\mathrm{PE}$ for 2 cycles then EMA-CO re-induction. No serious side effects from chemotherapy were encountered in other patients.
From 74 patients, one who was in stage IV (ultra-HR) was still having ongoing treatment whereas 73 had completed their primary treatment. Total of $62 / 73$ patients including the 5 patients who did not have chemotherapy (84.9\%) or $57 / 68$ patients who had first-line chemotherapy (83.8\%) achieved remission. Eleven patients who had 8-day IM methotrexate-folinic acid (MTX-FA) showed poor response (10 patients with rising or plateau of B-hCG) or experienced side effects from MTX-FA (1 patient) had second-line drugs. All of them were in LR group, with 7 (63.6\%) had scores 5-6 and 4 (36.4) had score 1-4. No significant differences of index pregnancy or stage were found between the responders to first-line chemotherapy and non-responders (data not shown). Among these 11 patients, 3 did not respond to secondline chemotherapy. One patient underwent interval hysterectomy (choriocarcinoma pathology) and subsequent bilateral salpingooophorectomy (no specific pathology) before third-line chemotherapy. She still had progressive diseases (lung and brain), so had further-line chemotherapy with no response and finally succumbed to death. The other 2 had complete remission from the third-line drugs. Summary of chemotherapy treatment in the primary setting is shown in Table 4 and illustrated in Figure 1.

Among 73 patients who had complete remission from primary treatment, 5 patients $(6.8 \%)$ had recurrences. All were in stage I and LR. The median time to recur after previous treatment completion was 2.6 months (range, 1.6-26.9 months). All recurrences presented with serum B-hCG elevation; 3 also had new metastatic lung lesions. One had single lung lesion resection without chemotherapy whereas the other 4 had chemotherapy re-induction. Two had second recurrences. Characteristic features and treatment of 5 GTN patients with recurrences are shown in Table 5.

Except 1 patient who did not respond to many regimens of chemotherapy and died of progressive diseases in brain and lung at 15.1 months, the others were alive after a median follow-up of 22.7 months (range, 0.37-210.9 months).

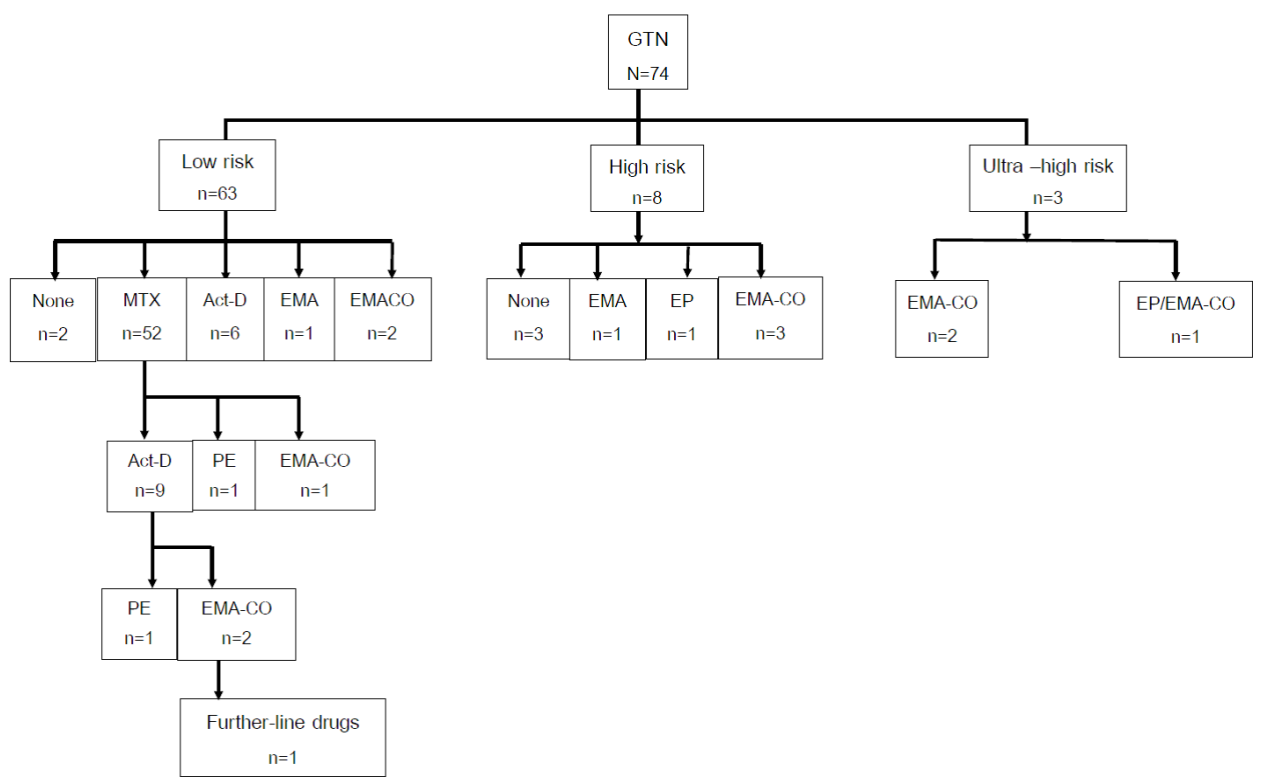

Figure 1. Chemotherapy given to gestational trophoblastic neoplasia in the primary setting $(\mathrm{N}=74)$ 
Tangiitgamol S (2020) Clinical Features, Treatment and Outcomes of Patients with Gestational Trophoblastic Neoplasm: An Experience from a Tertiary Center for Cancer Care in Thailand

Table 4. Chemotherapy used for gestational trophoblastic neoplasia in the primary treatment $(\mathrm{N}=74)$

\begin{tabular}{|c|c|c|c|c|c|c|c|}
\hline WHO risk group & No CMT & MTx & ACT-D & EMA & PE & EMA-CO & PE/ EMA-CO \\
\hline \multicolumn{8}{|l|}{ Low risk $(\mathrm{n}=63)$} \\
\hline First-line & $2(3.2)$ & $52(82.5)$ & $6(9.5)$ & $1(1.6)$ & - & $2(3.2)$ & - \\
\hline Second-line & $46(79.3)$ & - & $9(15.5)$ & - & $1(1.7)$ & $1(1.7)$ & - \\
\hline Third-line & - & - & - & - & $1(33.3)$ & $2(66.7)$ & - \\
\hline \multicolumn{8}{|l|}{ High risk $(\mathrm{n}=8)$} \\
\hline First line & $3(37.5)$ & - & - & $1(12.5)$ & $1(12.5)$ & $3(37.5)$ & - \\
\hline Second line & $8(100.0)$ & - & - & - & - & - & - \\
\hline \multicolumn{8}{|l|}{ Ultra HR (n=3) } \\
\hline First line & - & - & - & - & - & $2(66.7)$ & $1(33.3)$ \\
\hline Second line & $3(100.0)$ & - & - & - & - & - & - \\
\hline Third-line & - & - & - & - & - & - & - \\
\hline
\end{tabular}

Abbreviation: ACT-D, actinomycin-D; CMT, chemotherapy; EMA, etoposide, methotrexate, actinomycin;CO, cyclophosphamide, vincristine; MTx, methotrexate; PE, cisplatin, etoposide

Table 5. Characteristic features, treatment and outcomes of patients with recurrence gestation trophoblastic neoplasia

\begin{tabular}{|c|c|c|c|c|c|c|c|c|c|c|c|}
\hline \multirow[b]{2}{*}{ Patient } & \multirow[b]{2}{*}{ Age } & \multicolumn{3}{|c|}{ Primary setting } & \multicolumn{3}{|c|}{ First recurrence } & \multicolumn{3}{|c|}{ Second recurrence } & \multirow[b]{2}{*}{ OS, mos } \\
\hline & & Stage: Score & $\mathbf{R x} *$ cycle & DFI, mo & $\begin{array}{l}\text { Stage: } \\
\text { Score }\end{array}$ & $\mathbf{R x} *$ cylce & DFI, mo & $\begin{array}{l}\text { Stage: } \\
\text { Score }\end{array}$ & $\mathbf{R x} *$ cycle & DFI, mo & \\
\hline 1 & 38 & I: 0 & MTx*6 & 16.0 & III: 7 & Mass resection & 14.8 & - & - & - & 38.8 \\
\hline 2 & 49 & I: 5 & TAH only & 0.5 & III: 2 & MTx*6 & 38.8 & - & - & - & 44.4 \\
\hline 3 & 33 & I: 2 & $\mathrm{MTx}^{*} 10$ & 2.6 & I: 4 & ACT* 3 & 94.6 & - & - & - & 104.1 \\
\hline 4 & 30 & I: 2 & MTx*7 & 26.9 & III: 7 & EMACO*4 & 5.2 & III: 9 & TAH/ EMACO $* 4$ & 5.0 & 38.1 \\
\hline 5 & 30 & I: 1 & MTx*7 & 1.6 & I: 3 & $\mathrm{ACT}^{2} 4$ & 11.6 & I: 8 & TAH only & 86.2 & 116.8 \\
\hline
\end{tabular}

Abbreviation: ACT, actinomycin; DFI, disease-free interval; EMACO; etoposide, methotrexate, actinomycin, cyclcophosphamide, vincristine; MTx, methotrexate; TAH, total abdominal hysterectomy

Note: Patient\#4 had re-elevation of B-hCG and re-appearance of lung lesions after completion of chemotherapy for the first recurrence had hysterectomy (no specific pathology) followed by chemotherapy re-induction before remission. Patient \#5 had B-hCG elevation after completion of chemotherapy for the first recurrence, underwent hysterectomy (choriocarcinoma pathology) and was in remission without chemotherapy.

\section{Discussion}

The incidence of GTN was difficult to be compared across studies because various sources of information: population- or hospitaldatabase and different periods of study. Nevertheless, it appeared that GTN was more common in South America or Asia than Europe. The incidence of GTN per 10,000 pregnancies were reported ranging from 1.0 from a tertiary hospital-based study from Thailand [14] or $3.0 \%$ from population-based study from Korea [15] and only 0.3 to 0.4 from population-based studies from Sweden [16] or Netherland [17]. Unfortunately, the precise incidence or prevalence of GTN was not stated in this study because we were aware of incomplete data to verify all pregnancy diagnosis.

Some authors made the diagnosis of invasive mole based on the pattern of B-hCG regression curve [18]. In contrast, our study made a diagnosis of invasive mole only by pathologic findings from hysterectomy, or else they were simply diagnosed as GTN by failure to have B-hCG normalization (either rising or plateau) according to the WHO criteria. We were aware of expert opinions of Kohorn who stated that the staging and scoring of invasive mole may not follow those of FIGO and WHO respectively [19], so data of invasive mole were presented discretely from the other GTN patients whenever possible in our study.

Our study found $84 \%$ of our GTN patients had preceding molar pregnancy. This was higher than previous studies from the West which reported $30-50 \%$ of their GTN followed molar pregnancy $[2,20]$. Our data were similar to one report from Egypt which found 83\% molar pregnancy as preceding pregnancy [21]. We also found majority of our patients had early GTN diagnosis $<4$ months from preceding pregnancy (91\%), had early stage I (73\%), and were classified as low risk (85\%). Although only $15 \%$ of our patients were in HR group, $27 \%$ of all patients had metastatic diseases. On the other hand, 30\% of patients with metastatic diseases were in HR group (Table 3). This 30\% was similar to $32 \%$ HR metastatic group reported from one previous study [21]. These reflected that both risk group and sites of lesions (stage or metastasis) were important in comparing data across studies aside from being indicators for management and follow-up of an individuals.

Our staging investigations revealed that $34 \%$ did not have evidence of lesions anywhere but only abnormal B-hCG level. Nearly $40 \%$ had suspicious intrauterine lesions. Some authors performed uterine recurettage which resulted in fewer courses of chemotherapy required $[7,22]$. Our institution did not include this procedure for GTN management because it had no impact on treatment planning especially when we were aware of possible complications, such as, bleeding or uterine perforation. Hysterectomy was, instead, preferred and performed in $18 \%$ in our study. Except one patient who had hysterectomy (despite her young age) due to uterine perforation, the other patients who had hysterectomy were older, had high risk score, tended to have limited disease in the uterus, or had poor response to chemotherapy. The rates and indications of hysterectomy in our study were similar to $17-18 \%$ rates of hysterectomy in other reports $[3,4]$. The indications for surgery in previous studies were older age, high risk score or chemotherapy-resistant tumor $[3,5,23,24]$ as well as profuse vaginal bleeding or uterine perforation $[3,5,6,25,26]$. Although 
few studies demonstrated significantly shorter treatment duration with fewer chemotherapy cycles $[3,27$ ) or some $(13 \%$ to $42 \%)$ even achieved remission after hysterectomy $[21,28]$. Our study did not find significant benefit of hysterectomy on number of chemotherapy cycles. Nevertheless, we found 4 out of 13 patients who had hysterectomy (30.8\%) spontaneously had B-hCG declined after hysterectomy without chemotherapy.

Chemotherapeutic regimens given to GTN patients are generally determined by their risk: single agents for LR and multi-agents for HR and ultra-HR diseases (FIGO). Methotrexate and actinomycin were the 2 most common single agent in LR group. Some used intravenous actinomycin $[14,29]$ whereas our institution preferred intramuscular 8-day MTX-FA because of the more convenient route of administration than 5-day IV dactinomycin. Approximately $84 \%$ of patients had complete remission with single first-line chemotherapy in our study. We could not demonstrate any features could significantly predict failure from first-line treatment. Although all patients who required secondline chemotherapy had received MTX-FA (11/52 patients or $21.2 \%$ ) compared to none who had dactinomycin ( $0 / 6$ patients), the numbers of patients in each group were too small to make a definite comment on their efficacy. A systematic review and meta-analysis which reported almost 4 folds treatment failure from weekly methotrexate compared to pulsed dactinomycin [11]. However, data of significant lower efficacy of 8 -day MTX-FA than dactinomycin ( $74 \%$ vs $100 \%$ complete remission) were reported only in one small trial (with 49 participants) by Lertkhachonsuk et al. [30]. The results from an ongoing phase III trial of the Gynecologic Oncology Group [31] comparing the 2 regimens would provide more data from in this low risk group GTN.

Aside from the impact of chemotherapy regimen, other studies demonstrated that high pretreatment hCG, interval from previous pregnancy more than 7 months, tumor size of over $5 \mathrm{~cm}$, or FIGO score $\geq 5$ were predictive of single-agent resistance [3,29]. Only higher number of failures had scores 5-6 than scores 1-4 was observed in our study. However, definite conclusion from our findings could not be made due to small number of patients with this event especially when all failures were in LR group.

Our deviation from the FIGO recommendation was that $4 \%$ of our patients had combined therapy despite being in LR group. They were either referred from elsewhere or treated by a medical oncologist outside gynecologic oncology service. This might reflect the situation in real clinical practice wherein the physician's judgment and experience had influence his medical care for GTN patients. Although no serious adverse events were encountered, we would like to emphasize medical care by experienced gynecologic oncologist especially in a team approach manner to select the most appropriate treatment for these GTN patients.

High remission rate of $85 \%$ was observed in our study. Effective chemotherapy had led to excellent survival outcome even in those who failed first-line drug or those who had recurrences. Salvage chemotherapy or surgery could successfully induce remission. Data from previous studies reported survival ranging from 59\% in the remote past to $93 \%$ or $95 \%$ during later years $[21,32]$, or $78 \%$ in HR metastatic diseases [24]. Some poor prognostic factors, such as, presence of brain and/or liver metastases, resistance to combination chemotherapy and the type of index pregnancy were reported. With a small number of patients and events found in our study, data of prognostic factors could not be explored in detail. Nevertheless, few poor features were observed: the patients with scores 5-6 had higher failure than those with scores $<4$ and the only one death in our study had chemoresistant tumor with brain metastasis which should be signs of dismal prognosis.

In conclusion, our study found majority of our GTN patients had preceding molar pregnancy and short interval $<4$ months before a diagnosis of GTN. Factors associated with HR or ultra-HR were nonmolar index pregnancy, interval $\geq 4$ months, and FIGO stage III-IV. Surgical treatment may be a curative treatment in some patients even in HR group. Survival outcome was excellent even with recurrences.

\section{Acknowledgement}

The authors thank the officers in gynecologic oncology ward for their administrative support in acquiring all the patient's charts for data collection.

\section{Ethical approval}

The study met criteria for a waiver of inform consent from an individuals. The study was conducted after an approval from the Ethical Committees of the institution. (COA 181/61).

\section{Contribution}

ST: Project development, data collection, data analysis, manuscript writing/editing/ approval.

SS: Project development, data collection, manuscript editing/ approval.

\section{Funding}

This work was granted by Navamindradhiraj Research Fund for the study connduct and by the Faculty of Medicine Vajira Hospital Facilitating Research Fund for manuscript preparation and publication.

\section{Conflict of interest}

All authors declare no conflict of interest.

\section{References}

1. Hui P, Baergen R, Cheung ANY, Fukunaga M, Gersell D, et al. Chapter 6: Gestationa trophoblastic disease. In: Robert J. Kurman, Maria Luisa Carcangiu, C. Simon Herrington, Robert H. Young, (Eds.): WHO Classification of Tumours of Female Reproductive Organs. IARC: Lyon 2014, p.156-67.

2. Ngan HYS, Seckl MJ, Berkowitz RS, Xiang Y, Golfier F, et al. (2018) Update on the diagnosis and management of gestational trophoblastic disease. Int J Gynecol Obstet 143: 79-85. [Crossref]

3. Eysbouts YK, Massuger LFAG, IntHout J, Lok CAR, Sweep FCGJ, et al. (2017) The added value of hysterectomy in the management of gestational trophoblastic neoplasia. Gynecol Oncol 145: 536-542. [Crossref]

4. Pires LV, Uberti EM, Fajardo Mdo C, da Cunha AG, Rosa MW, et al. (2012) Role of hysterectomy in the management of patients with gestational trophoblasticneoplasia: importance of receiving treatment in reference centers. J Reprod Med 57: 359-368. [Crossref]

5. Fulop V, Szigetvari I, Szepesi J, Vegh G, Zsirai L, et al. (2016) Role of hysterectomy in the management of patients with gestational trophoblastic neoplasia: importance of receiving treatment in reference centers. J Reprod Med 61: 197-204. [Crossref]

6. Pisal N, North C, Tidy J, Hancock B (2002) Role of hysterectomy in management of gestational trophoblastic disease. Gynecol Oncol 87: 190-192. [Crossref]

7. Hemida RA, Toson E, Van Doorn HC (2011) The impact of uterine re-curettage, preevacuation and week-one level of hCG on the number of chemotherapy courses in treatment of post molar GTN. J Exp Ther Oncol 9: 217-220. [Crossref]

8. Braga A, Torres B, Burlá M, Maestá I, Sun SY, et al (2016) Is chemotherapy necessary for patients with molar pregnancy and human chorionic gonadotropin serum levels raised but falling at 6months after uterine evacuation? Gynecol Oncol 143: 558-564. [Crossref] 
9. Braga A, Biscaro A, do Amaral Giordani JM, Viggiano M, Elias KM, et al. (2018) Does a human chorionic gonadotropin level of over 20,000 IU/L four weeks after uterine evacuation for complete hydatidiform mole constitute an indication for chemotherapy for gestational trophoblastic neoplasia? Eur J Obstet Gynecol Reprod Biol 223: 50-55. [Crossref]

10. Lawrie TA, Alazzam M, Tidy J, Hancock BW, Osborne R (2016) First-line chemotherapy in low-risk gestational trophoblastic neoplasia. Cochrane Database Syst Rev: CD007102. [Crossref]

11. Alazzam M, Tidy J, Osborne R, Coleman R, Hancock BW, et al. (2016) Chemotherapy for resistant or recurrent gestational trophoblastic neoplasia. Cochrane Database Syst Rev: CD008891. [Crossref]

12. Ngan HY, Bender H, Benedet JL, Jones H, Montruccoli GC, et al. (2003) Gestational trophoblastic neoplasia, FIGO 2000 staging and classification. Int J Gynecol Obstet 83: 175-177. [Crossref]

13. Ngan HY, Seckl MJ, Berkowitz RS, Xiang Y, Golfier F, et al. (2015) Update on the diagnosis and management of gestational trophoblastic disease. Int J Gynaecol Obstet 131 Suppl 2: S123-S126. [Crossref]

14. Lertkhachonsuk R, Wairachpanich V (2016) Treatment outcomes of gestationa trophoblastic neoplasia in king chulalongkorn memorial hospital over two decades. $J$ Reprod Med 61: 238-242. [Crossref]

15. Yuk JS, Baek JC, Park JE, Jo HC, Park JK, et al. (2019) Incidence of gestational trophoblastic disease in South Korea: a longitudinal, population-based study. PeerJ 7 : e6490. [Crossref]

16. Eysbouts YK, Bulten J, Ottevanger PB, Thomas CMG, Ten Kate-Booij MJ, et al (2016) Trends in incidence for gestational trophoblastic disease over the last 20 years in a population-based study. Gynecol Oncol 140: 70-75. [Crossref]

17. Ringertz N (1970) Hydatidiform mole, invasive mole and choriocarcinoma in Sweden 1958-1965. Acta Obstet Gynecol Scand 49: 195-203. [Crossref]

18. Hando T, Ohno M, Kurose T (1998) Recent aspects of gestational trophoblastic disease in Japan. Int J Hynecol Obstet 60: S71-S76. [Crossref]

19. Kohorn EI (2007) Dynamic staging and risk factor scoring for gestational trophoblastic disease. Int J Gynecol Cancer 17: 1124-1130. [Crossref]

20. Mangili G, Lorusso D, Brown J, Pfisterer J, Massuger L, et al. (2014) Trophoblastic disease review for diagnosis and management: a joint report from the International Society for the Study of Trophoblastic Disease, European Organisation for the Treatment of Trophoblastic Disease, and the Gynecologic Cancer Inter Group. Int $J$ Gynecol Cancer 24: S109-S116. [Crossref]
21. El-Lamie IK, Shehata NA, Abou-Loz SK, Ei-Lamie KI (2000) Experience of the Gynecologic Oncology Unit at Ain Shams University in the treatment of gestational trophoblastic tumors. Int J Gynecol Cancer 10: 488-496. [Crossref]

22. Eoh KJ, Chung YS, Yim GW, Nam EJ, Kim S, et al. (2015) Role of surgical therapy in the management of gestational trophoblastic neoplasia. Obstet Gynecol Sci 58: 277 283. [Crossref]

23. Ghaemmaghami F, Ashrafgangooei T, Gillani MM, Mosavi A, Behtash N (2011) Majo surgeries performed for gestational trophoblastic neoplasm in a teaching hospital in Tehran, Iran. J Gynecol Oncol 22: 97-102. [Crossref]

24. Suzuka K, Matsui H, Iitsuka Y, Yamazawa K, Seki K, et al. (2001) Adjuvan hysterectomy in low-risk gestational trophoblastic disease. Obstet Gynecol 97: 431434. [Crossref]

25. Lehman E, Gershenson DM, Burke TW, Levenback C, Silva EG, et al. (1994) Salvage surgery for chemorefractory gestational trophoblastic disease. J Clin Oncol 12: 2737 2742. [Crossref]

26. El-Lamie IK, El Sayed HM, Badawie AG, Bayomi WA, El-Ghazaly HA, et al. (2006) Evolution of treatment of high-risk metastatic gestational trophoblastic tumors: Ain Shams University experience. Int J Gynecol Cancer 16: 866-874. [Crossref]

27. Clark RM, Nevadunsky NS, Ghosh S, Goldstein DP, Berkowitz RS (2010) The evolving role of hysterectomy in gestational trophoblastic neoplasia at the New England Trophoblastic Disease Center. J Repro Med 55: 194-198.

28. Cagayan SFS, Gacoba MCC (2006) Chemotherapy regimens used in the treatment of gestational trophoblastic neoplasia at Philippine General Hospital. J Reprod Med 71: 907-918

29. Lertkhachonsuk AA, Israngura N, Wilailak S, Tangtrakul S (2009) Actinomycin $\mathrm{d}$ versus methotrexate-folinic acid as the treatment of stage I, low-risk gestationa trophoblastic neoplasia: a randomized controlled trial. Int J Gynecol Cancer 19: 985 988

30. NCT01535053. A phase III randomized trial of pulse actinomycin-D versus multiday methotrexate for the treatment of low-risk gestational trophoblastic neoplasia. Available at https://clinicaltrials.gov/ct2/show/NCT01535053. Accessed on September 3rd, 2019.

31. Li L, Wan X, Feng F, Ren T, Yang J, et al. (2018) Pulse actinomycin D as first-line treatment of low-risk post-molar non-choriocarcinoma gestational trophoblastic neoplasia. BMC Cancer 18: 585. [Crossref]

32. Lurain JR, Hoekstra AV, Schink JC (2008) Results of treatment of patients with gestational trophoblastic neoplasia referred to the Brewer Trophoblastic Disease Cente after failure of treatment elsewhere (1979-2006). J Reprod Med 53: 535-540. [Crossref]

Copyright: (C2020 Tangjitgamol S. This is an open-access article distributed under the terms of the Creative Commons Attribution License, which permits unrestricted use, distribution, and reproduction in any medium, provided the original author and source are credited. 